\title{
The impact of terrestrial protected areas on vegetation extent and condition: a systematic review protocol
}

\author{
Roshan Sharma ${ }^{1,2^{*}} \mathbb{D}$, Johanna Eklund ${ }^{3}$, Megan Barnes ${ }^{4}$, Jonas Geldmannn ${ }^{5,9}$, Judith Schleicher ${ }^{6}$, \\ Robert L. Pressey ${ }^{7}$, Marco Gutierrez ${ }^{1}$, Simon Jones ${ }^{8}$ and Ascelin Gordon ${ }^{1}$
}

\begin{abstract}
Background: Establishing protected areas is a key approach to protecting nature. However, protected areas are often biased towards remote and less productive lands. It is important to evaluate the impacts protected areas have had, or in other words, what changes in outcomes of interest are attributable to protected areas. Studies that evaluate the impact of protected areas on vegetation - the state and processes that support biodiversity - are scarce and published in a range of disciplines. This systematic review will scope, identify, and synthesize studies that quantitatively measure the impact of protected areas on vegetation extent and condition. The findings will be useful for researchers and policy makers and provide important knowledge for setting post 2020 targets of the Convention on Biological Diversity. This review will also identify research gaps in the current evidence base and provide direction for future research.

Methods: This review follows the Collaboration for Environmental Evidence guidelines for evidence synthesis and complies with the ROSES (RepOrting standards for Systematic Evidence Synthesis) reporting framework. We will use a comprehensive search strategy developed through several rounds of scoping review to cover databases; Web of Science, Scopus and CAB Abstracts, 16 organizational websites, google scholar and existing review documents. Our search terms and strategies aim to find impact evaluation studies (both peer-reviewed and grey literature) in English from protected areas globally. The search results will be screened at title, abstract, and then full text by two independent reviewers. A quality appraisal of evidence will be conducted using Joanna Briggs Institute (JBI) risk of bias tool. Review results will be presented in the form of narrative synthesis, as well as in meta-analysis form, where data quality and amount allow.
\end{abstract}

Keywords: Evidence synthesis, Evaluation, Counterfactual, Deforestation, Degradation

\section{Background}

Protected areas (PAs) such as national parks, wildlife and nature reserves are one of the fundamental approaches to conserving nature by restricting human land and resource use [1]. IUCN defines PAs as "a clearly defined geographical space, recognized, dedicated, and managed,

\footnotetext{
*Correspondence: roshan.sharma@rmit.edu.au; roseanstore@hotmail.com ${ }^{1}$ Interdisciplinary Conservation Science Research Group, School of Global, Urban and Social Studies, RMIT University, Melbourne, Australia Full list of author information is available at the end of the article
}

through legal or other effective means, to achieve longterm conservation of nature with associated ecosystem services and cultural values" [2]. Since the campaign to expand PAs at the Worlds Park Congress in 1982, nations have worked to increase the area of land under protection. Currently, the global terrestrial PA estate has grown to cover more than 28.4 million square kilometres or 13 percent of the Earth's land surface [3]. Further, the Convention on Biological Diversity (CBD) has called to

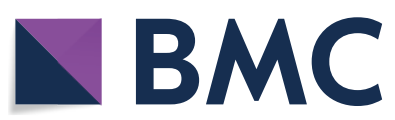

(c) The Author(s) 2020. This article is licensed under a Creative Commons Attribution 4.0 International License, which permits use, sharing, adaptation, distribution and reproduction in any medium or format, as long as you give appropriate credit to the original author(s) and the source, provide a link to the Creative Commons licence, and indicate if changes were made. The images or other third party material in this article are included in the article's Creative Commons licence, unless indicated otherwise in a credit line to the material. If material is not included in the article's Creative Commons licence and your intended use is not permitted by statutory regulation or exceeds the permitted use, you will need to obtain permission directly from the copyright holder. To view a copy of this licence, visit http://creativeco mmons.org/licenses/by/4.0/. The Creative Commons Public Domain Dedication waiver (http://creativecommons.org/publicdomain/ zero/1.0/) applies to the data made available in this article, unless otherwise stated in a credit line to the data. 
increase the protection to cover 17 percent (Aichi Target 11) of the land surface by 2020 .

This target represents a growing recognition that PAs are central to safeguard remaining habitats and species. Despite the increasing extent of the PA network and other conservation efforts [4], vegetation loss continues even within PAs [5]. Forests, in which $80 \%$ of the world's known terrestrial animals and plants are found [6], continue to decline globally [7]. Similarly, grasslands in biological hotspots are being converted [8]. Conversion is mainly led by expansion of croplands and urban areas, infrastructure, logging, mining, and fire [9]. Although PAs are a widely promoted instrument in conservation, there is criticism regarding the impact PAs are having on halting loss of biodiversity [10] and regarding the social and economic impacts they have on local people [11]. For instance, PAs are disproportionately established in areas that are unimportant for biodiversity [10] and are located in marginal lands with least pressure of conversion [12]. This means that PAs tend to be located in areas with steeper slopes, higher elevations, less productive land, and greater distance from roads and cities [12, 13].

Recent studies have estimated the impact of PAs in reducing vegetation loss (see these reviews $[14,15]$ ). While several studies have found PAs to be effective in reducing deforestation, others have found insignificant or negative impacts [15]. However, these results could be biased by over or under estimation if confounding factors that affect both the location of the PA and the probability of the outcome of the intervention are not considered. Other factors like spill over effects could also bias the evaluation [16]. Additionally, the non-random location of PA [e.g. 10,11] means that studies that do not account for location-bias fail to establish a credible counterfactual-what would have happened without the PAs. For instance, one example of a study design that is often inappropriate is undertaking an inside-outside comparison of a PA without establishing appropriate comparison groups. Such naïve evaluations can be misleading, because they do not account for confounding influences. Building a body of evidence on protected area effectiveness on reducing vegetation loss based on robust methods is therefore an important public good.

There have been several previous reviews in assessing protected area impacts. A systematic review similar in scope to what we propose here was published in 2013 by Geldmann et al. [15]. However, our review will be an important extension of work by Geldmann et al. for two reasons. Firstly, Geldmann et al. only reviewed studies up until 2010, and given the increasing interest on impact evaluation in the environment and conservation domains since 2010, there could be a large body of additional literature that should now be reviewed. Secondly, Geldmann et al, focused on only forests, while we broaden our search criteria to capture additional studies that focus on other ecosystem types such as grasslands and shrublands, and propose undertaking a quality appraisal of the final selected studies. Another review was published in 2018 by Schleicher et al, but was also limited only to forests and had narrow search criteria that only included studies from South America [14]. Puri et al. published a systematic map-an evidence gap map that doesn't provide a quantitative synthesis of effect and limiting to forest ecosystems in low and middle income countries [17].

During the formulation of review question, an expert team was established and consulted. This team included experts in terrestrial ecosystems and PAs (RLP, MB, AG, SJ, JE, JS), including those who have conducted research on the views of PA practitioners (JS, JE), and those who have prior experience in synthesizing studies on PA impacts (RLP, MB, JG, JS, JE). The collective experience and contacts of the team have allowed consultation with a wide variety of stakeholders including both academics and practitioners who gave feedback in our study design. Further, we consulted with several other academic experts from University of Melbourne, Interdisciplinary Conservation Science Research Group at RMIT University, WWF Nepal and India, and Centre for International Forestry Research (CIFOR) to ensure that the primary question was both answerable and relevant. These consultations contributed in defining the research questions, refining the scope of this review, and developing a comprehensive search strategy. Two librarians from RMIT University provided support in finalizing the search strategies.

By following the guidelines for the standard systematic review protocol for conservation evidence [18] and the ROSES reporting framework [19], we aim to collect and synthesize the published literature in English that uses appropriate methods for quantitative impact evaluation of PAs. By setting a strict impact evaluation criterion (see comparison terms used in Boolean search as described below) in initial search, dual screening, and critical quality appraisal in the full review phase, we aim to eliminate studies that do not follow a generally robust methodology (studies that don't have some measures to control for bias); thereby analysing studies on PA impact that do not contain significant bias or errors. We argue that including studies without robust study methods could provide biased results, and therefore mislead researchers, policy makers, and managers.

We hope that this review will synthesize scientific results that will help to inform researchers and policy makers on the efficacy of PAs in reducing biodiversity loss [20], and identify current trends and gaps in research on impact evaluations of PAs. Such information is key for the 
development of new post-2020 targets for the CBD. The debate is currently ongoing as to whether the focus of a successor to Aichi Target 11 should be on setting higher coverage targets for PAs, or, alternatively, emphasize outcome and impact measures [21-23]. This study will be a valuable contribution, synthesizing the state of the current knowledge of PA impacts.

\section{Objective of the review}

The objective of this systematic review is to systematically review and synthesize studies that evaluate the impact of PAs (intervention) on changes in vegetation extent and condition (outcomes). The outcome includes both the averted loss and degradation, and gain and restoration of vegetation. The review will focus on terrestrial ecosystems (population or subjects), and excludes marine, intertidal and unclassified areas. In this study, vegetation means assemblage of plant species within a biome such as forests, grasslands, and shrublands. The systematic review is built on the elements based on PICO (Population, Intervention, Comparator, and Outcomes). The details of PICO elements are provided in Additional file 2. By building a systematic review we will address the following research question: What is the impact of protected areas on vegetation extent and condition?

The evidence base will be analysed and categorized using a pre-developed data extraction framework described below to answer the following secondary questions:

(i) What is the state of the evidence-number of studies, study location, protection period, intervention type (type/category of protected area)?

(ii) What type of study designs have been used in studies, and what are their strengths and limitations?

(iii) What are the data types and sources used by the studies and are they appropriate?

(iv) What are the covariates used as confounding factors in each study?

(v) What outcomes are measured and how are they measured?

(vi) How do the impacts found in our review vary across biogeographic and political scales?

(vii) What are the trends in the estimated counterfactuals for protected areas, and how do they vary across biogeographic and political scales?

(viii) What are the trends of the number of studies and quality of evidence over time?

(ix) What are the major gaps in the evidence base?

\section{Methods}

This review follows the Collaboration for Environmental Evidence guidelines for evidence synthesis and complies with the ROSES (RepOrting standards for Systematic
Evidence Synthesis) reporting framework (see Additional file 1). The methods for building the evidence include the following steps: (i) development of search keywords (ii) searching literature in various database, search engines, and searching the citations in found papers, (iii) screening of articles using eligibility criteria to develop a list of articles. Using the selected papers, the following two steps will be undertaken (iv) critical appraisal of the selected articles and preparation of final list, (v) extracting data to be used to answer the research questions, and (vi) synthesis of the findings.

The search strategy is designed to retrieve all publications on protected area impact on vegetation. We will retrieve both 'peer-reviewed' and a limited selection of published and unpublished documents (e.g. conference papers, academic and organizational reports, and book chapters) that were not submitted to peer-reviewed journals, more generally referred to as 'gray literature'. Including gray literature also helps to extract relevant information such as the details of the methods used in evaluation and contributes to minimizing publication bias [24]. To maximize our search and minimize the risk of publication bias, we will search the published and unpublished literature from the following sources: peerreviewed databases, search engines, searching within papers to find other papers, and a targeted search on the websites of conservation agencies and research centres. Searching several sources is intended to help to maximize the relevant articles captured in our review [25]. Selection of these sources was based on previous systematic reviews and maps on related topics $[26,27]$.

\section{Searching for articles \\ Search languages}

We will review only English language publications. A caveat to this is that if there are publications in other languages from regions particularly from South America and Asia, these will be excluded from the review. We acknowledge that excluding literature written in other languages is a shortcoming. However, we do not have the resources needed to work in other languages, especially when we have dual consistency checking in screening, full review, quality appraisal and data extraction.

\section{Search strings}

A search string based on PICO (Population or Subject, Intervention, Comparator, and Outcome) [28] related keywords in English will be used to query publication databases, online repositories, and search engines. Literature review and consultation with experts helped in developing the search strings. The team worked with two librarians-search specialists at RMIT University to develop a comprehensive search strategy. The keywords 
combination and Boolean operators are based on a search in Web of Science. The search string might be further refined during implementation of the full search. The detailed definition of PICO elements, process for developing the search keywords, and the final search strings is provided (see Additional file 2). We compared the search results against a list of benchmark studies to check the effectiveness of the keywords. The list of benchmark studies is also provided (see Additional file 2: Table S3).

\section{Publication databases to be searched}

Web of Science (http://apps.webofknowledge.com/), Scopus (http://scopus.com/), and CAB Abstracts (https ://www.cabi.org/publishing-products/cab-abstracts/)search platforms and peer-reviewed databases that cover natural and social sciences-will be accessed and searched through RMIT University and University of Helsinki. The search string will be used to search each database by topic terms, which includes title, abstract and keywords. As these databases have different structures, the search string developed for Web of Science (see Additional file 2) will need to be modified for Scopus and $\mathrm{CAB}$ Abstracts. The results from these databases will be combined, and duplicates will be removed in Endnote X9 [29].

\section{Internet searches to be conducted}

A web-based search engine, Google Scholar (http://schol ar.google.com) will also be used to search for both 'peerreviewed' and 'grey literature' (articles, books, theses, and reports). Google scholar supplements bibliographic searches and can increase the comprehensiveness of the search [30]. Because we aim to conduct a quick search on title of publications, only a subset of terms "protected areas", "impact evaluation", "counterfactual", "evidence", "deforestation", and "vegetation" will be used. Only a title search will be conducted because searching by title is more efficient than searching the full text [30]. We will use the Publish or Perish software [31] to download the first 1000 results from Google Scholar. Relevant articles returned by the software will be added to the reference list in Endnote.

\section{Specialist searches}

A targeted search of the websites of specialist organizations in the conservation and development sectors will be conducted to identify additional grey literature. We will hand search every organizational website. The list of websites of different conservation-oriented organizations is provided as an Additional file 3.

\section{Supplementary searches}

The reference lists in the articles selected for full review and other review articles will be used to supplement the search. First, we will generate a list of all the unique references from the articles selected for full review. From this list we will remove the articles that were included and screened in the preliminary search from Web of Science and Scopus. We will undertake the same screening process for the remaining articles and if relevant, we add them to be included in the full review. In our view, this may be an important way to access relevant literature that would not be accessible by the search strategies proposed above.

\section{Search comprehensiveness}

The search strategy in our review is largely dependent on English language literature and online search platforms and peer-reviewed and non-peer reviewed databases. Web of Science, Scopus, CAB Abstracts cover articles from natural sciences including major conservation, environment, and development journals. Further, search comprehensiveness was tested by comparing the search results to a list of benchmark studies. Because the databases only include articles published from 1980 onwards, those that are published before that period will be not be captured by the search. Literature on languages other than English will also not be included due to limitations in resources. Some of these constraints are addressed by using a variety of approaches such as searching in Google scholar and targeted search. Checking reference list of selected papers and review papers will also ensure that papers are not missed out. Automated alert of any papers published that meet the initial search strategy will be set up in bibliographic databases so any new papers that become available while the analysis is being undertaken can be included in the review.

\section{Article screening and study eligibility criteria} Screening process

Citations identified from the databases will be imported into the reference management software Endnote X9 [29] and duplicates will be removed. The citations will be imported into the Covidence systematic review software (https://www.covidence.org/home). At the first stage, two reviewers (RS and MG) will independently screen both titles and abstracts according to the eligibility criteria (see Table 1). A third reviewer (AG) will help resolve any disagreements between RS and MG. During the second stage, full texts of potentially relevant articles will be retrieved and again screened by two reviewers (RS and JE) independently using a set of eligibility criteria (see Table 1). All disagreement concerning the inclusion or the exclusion of articles (including the articles authored 
Table 1 Inclusion and exclusion criteria for full-review screening

\begin{tabular}{|c|c|c|}
\hline & Inclusion criteria & Exclusion criteria \\
\hline Population & Terrestrial ecosystems & Freshwater, marine, and coastal ecosystems \\
\hline Intervention & $\begin{array}{l}\text { All types of protected areas (both IUCN categories, and others } \\
\text { with formal or informal designations) }\end{array}$ & $\begin{array}{l}\text { Other conservation interventions such as payment for ecosystem } \\
\text { service interventions, REDD+ }\end{array}$ \\
\hline Outcomes & $\begin{array}{l}\text { Studies that quantitatively measure the biophysical outcomes of } \\
\text { change in vegetation extent and condition }\end{array}$ & $\begin{array}{l}\text { Studies that do not quantitatively measure biophysical outcomes of } \\
\text { change in vegetation extent and condition }\end{array}$ \\
\hline Study designs & $\begin{array}{l}\text { Study that have comparison groups to measure impact. Only } \\
\text { experimental and quasi-experimental designs (e.g. randomized } \\
\text { controlled trials (RCTs), difference-in-difference (DID), matching, } \\
\text { regression discontinuity designs (RDD), instrumental variables } \\
\text { (IV) }\end{array}$ & $\begin{array}{l}\text { Personal views and perspectives, case studies, theoretical studies } \\
\text { and models. Studies using observational data with no controls, } \\
\text { correlation studies }\end{array}$ \\
\hline
\end{tabular}

by the reviewers) will be made after a discussion with the review team. Papers on systematic review and meta-analysis will be included as separate groups. The list of articles excluded in the full text review, along with reasons for exclusion will be provided in a separate table.

\section{Eligibility criteria}

See Table 1.

\section{Study validity assessment}

The articles that have been selected after the full text review will be critically appraised following the Collaboration for Environmental Evidence guidelines [18]. Use of formal validity assessment tools or instruments over expert judgements is recommended by various organizations that produce systematic reviews and other types of evidence synthesis such as the Cochrane (for health sciences), Campbell Collaboration (for public policy), and the Collaboration for Environmental Evidence (for environmental science). We will use the Joanna Briggs Institute (JBI) quality appraisal tool for quasi-experimental study design [32], as it is more closely related to impact evaluation studies. This toolkit present variables that we consider critical in influencing the internal validity of study and focuses on selection bias, confounding bias, and measurement bias. The quality appraisal toolkit is provided as an additional file (see Additional file 4). Given that generalisability vary geographically and with many other factors, we will not assess the external validity (extent to which the study findings are generalisable). To maintain consistency, two reviewers will independently conduct quality appraisal of the selected studies. Although our search strategy will capture any studies that use true experimental designs (randomized control trials) to evaluate PAs, we are currently not aware of any studies, and anticipate there won't be any studies that use such designs in the case of PAs. If any studies that use such methods are found in the full search, we will modify and use the Joanna Briggs Institute (JBI) quality appraisal tool for randomized control trials.
Quality appraisal results for each study will be reported as a separate table. JBI quality appraisal toolkit has 9 categories, if a study is appraised to have more than 5 biases they will be categorized as high biased studies. Analysis with and without the high biased studies will be conducted to compare the results. An impact evaluation estimates the impact by comparing the outcomes for an intervention group with the estimate of the counterfactual from a comparison group. We will exclude studies where only one group is observed (i.e. there is no comparison group) as it does not demonstrate cause. This refers to question number 4 in the JBI toolkit. The reason for exclusion will be provided in the table. If there is any study that gets selected in the full review phase and if any of the authors (who will conduct quality appraisal) are co-authors in that study, the study will be reviewed by other co-authors.

\section{Data coding and extraction strategy}

A standard questionnaire developed in spreadsheet form will be used to extract key information (including both qualitative and quantitative information) from the selected articles in the full article review. For each of the final list of studies, the following data will be extracted.

(i) Intervention/PA characteristics: category of PA, year of establishment, year of evaluation, size of PA, management and governance type, country of PA, socio-economic characteristics of country

(ii) Study characteristics: study design, data and method of evaluation (sample size, data type and source, resolution and extent of data, validity of data, confounders described and measured (covariates), and study biases reported

(iii) Outcome characteristics: category of outcomes (vegetation loss or gain, degradation or restoration) and their characteristics or meta-data (e.g. mean, standard deviation, standard error). 
If the reviewers feel any further data could be useful in the final review, we will add data to the final spreadsheet during the extraction phase. If needed, we will contact the authors through emails to obtain missing or other relevant information. To check consistency, data will be extracted by RS and MG independently. All extracted data records will be made available as additional files.

\section{Potential effect modifiers/reasons for heterogeneity}

To understand the variation in the PA outcomes we will identify and list the potential effect modifiers from the studies. There are several biogeographic, environmental and socio-economic factors that could result in the heterogeneity of impacts found in different studies. Some of the potential effect modifiers identified through author's experience and previous findings [33] that will be explored are: (i) category of PA, (ii) governance type of PA, (iii) location of PA (e.g. region, country, topographic features), (iv) size of PA, (v) date and period of establishment, (vi) socio-economic context of the state or country of PA (e.g. corruption index, gross domestic product, global peace index etc.), (vii), type of ecosystem, among others. This list is not exhaustive. A comprehensive list of effect modifiers will be presented in the systematic review. If information on modifiers is not present in the article itself, data will be supplemented from other sources like World Database on Protected Areas (WDPA) (https://www.protectedplanet.net/), World Bank, IUCN, and others. Sub-group analysis will be conducted in RevMan 5 [34] to analyse the effect of modifiers.

\section{Data synthesis and presentation}

The data synthesis will include both a narrative synthesis and a summary of findings (as descriptive statistics) of each study which will be included in a tabular form and recorded in an Excel spreadsheet. The spreadsheet will be available as a supplementary information of the systematic review. We anticipate that the studies in this review will vary widely in terms of socio-economic and environmental contexts and types of outcomes reported. Thus, at this stage it is unclear whether it is feasible to conduct a quantitative synthesis. If enough studies are found (that report effects or have the technical data to estimate effect size such as the ratios, mean and measures of variations) and if the variability among the studies are low, we will apply a random-effects model to synthesize effect size [35]. If the studies report continuous data types (means and standard deviations), mean difference or standardized mean difference will be used as a measure of effect. If the studies report proportion or percentage, risk difference will be used as a measure of effect [35]. An alpha level of 0.05 will be considered statistically significant. Potential publication bias will be checked by visual inspection of funnel plots [35]. We will examine the distribution of resulting effect sizes in the selected studies and explore the extent they vary with biogeographic, environmental and socio-economic factors described above. Meta-analysis will be conducted in RevMan 5 [34]. The review will report on what impact PAs have on vegetation extent and condition, data and methods used for impact evaluation, research gaps and the need and opportunities for future research.

\section{Supplementary information}

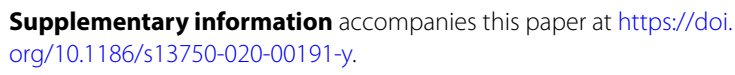

Additional file 1. Completed ROSES form.

Additional file 2. Final search strings, scoping exercise, and the list of benchmark studies.

Additional file 3. List of organizational websites.

Additional file 4. Quality appraisal toolkit.

\section{Acknowledgements}

This work was supported by an RMIT Research Stipend Scholarship (RRSS) and RMIT Research International Tuition Fee Scholarship (RRITFS). JE acknowledges the Kone foundation for funding. The authors would like to thank Ms Judy Maxwell and Ms Emma Rose at RMIT Carlton Library, Melbourne for their support in finalising the search strategies. The authors would also like to thank the experts, editors, and peer-reviewers whose comments and suggestions helped to improve the manuscript.

\section{Authors' contributions}

$R S, A G, S J, J E, J G, R L P$, and MB jointly conceived and designed the study. RS developed the first draft of the protocol. RS, JE, JG, JS, MB, AG, SJ developed the search strategy. JE, MB, JG, JS, RLP, SJ, and MG contributed to the revisions of the protocol. All authors provided critical comments for revision. All authors read and approved the final manuscript.

Funding

Not applicable.

Availability of data and materials

Not applicable

Ethics approval and consent to participate Not applicable.

Consent for publication

Not applicable.

Competing interests

The authors declare that they do not have competing interests.

\section{Author details}

${ }^{1}$ Interdisciplinary Conservation Science Research Group, School of Global, Urban and Social Studies, RMIT University, Melbourne, Australia. ${ }^{2}$ Global Institute of Interdisciplinary Studies, Kathmandu, Nepal. ${ }^{3}$ Department of Geosciences and Geography, University of Helsinki, Helsinki, Finland. ${ }^{4}$ Centre for Environmental Economics and Policy, The University of Western Australia, 35 Stirling Highway, Crawley, WA 6009, Australia. ${ }^{5}$ Department of Zoology, University of Cambridge, Cambridge, UK. ${ }^{6}$ Department of Geography, University of Cambridge, Cambridge, UK. ${ }^{7}$ Australian Research Council Centre of Excellence for Coral Reef Studies, James Cook University, Townville, Australia. ${ }^{8}$ School of Science, RMIT University, Melbourne, Australia. ${ }^{9}$ Center 
for Macroecology, Evolution and Climate, Globe institute, University of Copenhagen, Copenhagen, Denmark.

Received: 4 November 2019 Accepted: 24 April 2020

Published online: 29 April 2020

\section{References}

1. Juffe-Bignoli D, Burgess ND, Bingham H, Belle E, De Lima M, Deguignet M, et al. Protected Planet Report 2014. Cambridge: UNEP-WCMC; 2014.

2. Dudley N. Guidelines for applying protected area management categories. Gland: IUCN; 2008.

3. UNEP-WDPA. The world database on protected areas. 2019.

4. Watson JE, Dudley N, Segan DB, Hockings M. The performance and potential of protected areas. Nature. 2014;515(7525):67-73.

5. Clark NE, Boakes EH, McGowan PJ, Mace GM, Fuller RA. Protected areas in South Asia have not prevented habitat loss: a study using historical models of land-use change. PLOS ONE. 2013;8(5):e65298.

6. CBD and UNEP. "Forest-based solutions for accelerating achievement of the SDGs" thirteenth session of the United Nations Forum on Forests (UNFF13). Secretariat of the Convention on Biological Diversity and United Nations Environment Programme; 2018.

7. Hansen MC, Potapov PV, Moore R, Hancher M, Turubanova SA, Tyukavina $A$, et al. High-resolution global maps of 21st-century forest cover change. Science. 2013;342(6160):850-3.

8. Ceballos G, Davidson A, List R, Pacheco J, Manzano-Fischer P, SantosBarrera $G$, et al. Rapid decline of a grassland system and its ecological and conservation implications. PLoS ONE. 2010;5(1):e8562.

9. Curtis PG, Slay CM, Harris NL, Tyukavina A, Hansen MC. Classifying drivers of global forest loss. Science. 2018;361(6407):1108-11.

10. Venter O, Magrach A, Outram N, Klein CJ, Possingham HP, Di Marco M, et al. Bias in protected-area location and its effects on long-term aspirations of biodiversity conventions. Conserv Biol. 2018;32(1):127-34.

11. Schleicher J, Zaehringer JG, Fastré C, Vira B, Visconti P, Sandbrook C. Protecting half of the planet could directly affect over one billion people. Nat Sustain. 2019;2(12):1094-6.

12. Joppa LN, Pfaff A. High and far: biases in the location of protected areas. PLOS ONE. 2009:4(12):e8273.

13. Scott JM, Davis FW, McGhie RG, Wright RG, Groves C, Estes J. Nature reserves: do they capture the full range of America's biological diversity? Ecol Appl. 2001;11(4):999-1007.

14. Schleicher J. The environmental and social impacts of protected areas and conservation concessions in South America. Curr Opin Env Sust. 2018;32:1-8.

15. Geldmann J, Barnes M, Coad L, Craigie ID, Hockings M, Burgess ND. Effectiveness of terrestrial protected areas in reducing habitat loss and population declines. Biol Conserv. 2013;161:230-8.

16. Andam KS, Ferraro PJ, Pfaff A, Sanchez-Azofeifa GA, Robalino JA. Measuring the effectiveness of protected area networks in reducing deforestation. Proc Natl Acad Sci USA. 2008;105(42):16089-94.

17. Puri J, Nath M, Bhatia R, Glew L. Examining the evidence base for forest conservation interventions. International Initiative for Impact Evalutation (3ie) Evidence Gap Map Report. 2016;4:1-70.

18. Collaboration for Environmental Evidence. Guidelines and Standards for Evidence synthesis in Environmental Management. Version 5.0 (Pullin AS, Frampton GK, Livoreil B, Petrokofsky G, Eds) 2018 www.environmentalev idence.org/information-for-authors Accessed 2 July 2019.
19. Haddaway NR, Macura B, Whaley P, Pullin AS. ROSES RepOrting standards for Systematic Evidence Syntheses: pro forma, flow-diagram and descriptive summary of the plan and conduct of environmental systematic reviews and systematic maps. Environ Evid. 2018;7(1):7.

20. Dicks LV, Walsh JC, Sutherland WJ. Organising evidence for environmental management decisions: a'4S' hierarchy. Trends Ecol Evol. 2014;29(11):607-13.

21. Visconti P, Butchart SHM, Brooks TM, Langhammer PF, Marnewick D, Vergara S, et al. Protected area targets post-2020. Science. 2019;364(6437):239-41.

22. Visconti P, Butchart SHM, Brooks TM, Langhammer PF, Marnewick D, Vergara S, et al. A bold successor to Aichi Target 11-Response. Science. 2019:365(6454):650-1.

23. Woodley S, Baillie JEM, Dudley N, Hockings M, Kingston N, Laffoley D, et al. A bold successor to Aichi Target 11. Science. 2019;365(6454):649-50.

24. Hopewell S, McDonald S, Clarke M, Egger M. Grey literature in meta-analyses of randomized trials of health care interventions. Cochrane Database Syst Rev. 2007;18(2):MR000010.

25. Avenell A, Handoll HH, Grant AM. Lessons for search strategies from a systematic review, in The Cochrane Library, of nutritional supplementation trials in patients after hip fracture. Am J Clin Nutr. 2001;73(3):505-10.

26. McIntosh EJ, Chapman S, Kearney SG, Williams B, Althor G, Thorn JPR, et al. Absence of evidence for the conservation outcomes of systematic conservation planning around the globe: a systematic map. Environ Evid. 2018;7(1):22.

27. Bottrill M, Cheng S, Garside R, Wongbusarakum S, Roe D, Holland MB, et al. What are the impacts of nature conservation interventions on human well-being: a systematic map protocol. Environ Evid. 2014;3(1):16.

28. Moher D, Shamseer L, Clarke M, Ghersi D, Liberati A, Petticrew M, et al. Preferred reporting items for systematic review and meta-analysis protocols (PRISMA-P) 2015 statement. Syst Rev. 2015;4(1):1.

29. Moher D, Liberati A, Tetzlaff J, Altman DG, Group P. Preferred reporting items for systematic reviews and meta-analyses: the PRISMA statement. Int J Surg. 2010;8(5):336-41.

30. Haddaway NR, Collins AM, Coughlin D, Kirk S. The role of Google Scholar in evidence reviews and its applicability to grey literature searching. PLoS ONE. 2015;10(9):e0138237.

31. Harzing AW. Publish or Perish 2007. https://harzing.com/resources/publi sh-or-perish.

32. JBI. Critical Appraisal Tools: Joanna Briggs Institute, The University of Adelaide; 2019. https://joannabriggs.org/critical_appraisal_tools.

33. Busch J, Ferretti-Gallon K. What drives deforestation and what stops it? A meta-analysis. Rev Environ Econ Policy. 2017;11(1):3-23.

34. Review Manager (RevMan) [Computer Program] Version 5.3 Copenhagen: $=$ : The Nordic Cochrane Centre, The Cochrane Collaboration; 2014 https://community.cochrane.org/help/tools-and-software/revman-5.

35. Borenstein M, Hedges LV, Higgins JP, Rothstein HR. Introduction to metaanalysis. Hoboken: Wiley; 2011.

\section{Publisher's Note}

Springer Nature remains neutral with regard to jurisdictional claims in published maps and institutional affiliations.

Ready to submit your research? Choose BMC and benefit from:

- fast, convenient online submission

- thorough peer review by experienced researchers in your field

- rapid publication on acceptance

- support for research data, including large and complex data types

- gold Open Access which fosters wider collaboration and increased citations

- maximum visibility for your research: over 100M website views per year

At BMC, research is always in progress.

Learn more biomedcentral.com/submissions 